\title{
Evaluation of Thickness and Residual Stress of Shallow Surface Regions from Diffraction Profiles
}

\author{
Wulf Pfeiffer ${ }^{a^{*}}$ and Eduard Reisacher ${ }^{\mathrm{b}}$ \\ Fraunhofer Institute for Mechanics of Materials IWM, Wöhlerstr. 11, 79108 Freiburg, Germany \\ awulf.pfeiffer@iwm.fraunhofer.de*, beduard.reisacher@iwm.fraunhofer.de
}

Keywords: Residual Stresses, Stress Gradient, Diffraction Profile, Shot Peening, Ceramic

\begin{abstract}
Residual stresses resulting from surface treatment methods like shot peening generally show a more or less steep stress gradient versus depth. X-ray diffraction is the most used method for depth profiling in case of near-surface stress gradients due to the small penetration depth of X-rays resulting in a high depth resolution. When the stress gradient is very steep and the stressed surface layer is in the range of the penetration depth of the X-rays, asymmetric diffraction lines may occur and determination of the diffraction line position has to be done with great care. In case of the stressed surface layer being thinner than the penetration depth of the X-rays a (partial) splitting of the diffraction line may occur which may allow to separately calculate both the mean stresses of the layer and the base material and additionally to determine the thickness of the stressed surface layer. For a shot peened silicon nitride ceramic the evaluation of the mean stress in the thin surface layer, the stress in the underlying base material and the thickness of the stressed surface layer on basis of profile fitting methods is demonstrated with special emphasis on the influence of the method used for determining the diffraction line position.
\end{abstract}

\section{Introduction}

Residual stresses resulting from surface treatment methods like shot peening generally show a more or less steep stress gradient versus depth. X-ray diffraction is the most used method for depth profiling in case of near-surface stress gradients due to the small penetration depth of X-rays resulting in a high depth resolution. In case of steep stress gradients being in the range of the penetration depth of X-rays various methods for calculation of the stress-depth profiles from stresspenetration depth profiles have been presented $[1,2,3]$. Although it is now well known that such stress gradients may result in asymmetric diffraction lines $[4,5,6]$ little attention has been applied to the method to be used for the determination of the relevant diffraction angle from the intensity distribution of the diffraction line. Only the center of gravity of the complete diffraction line can be related to the depth inspected by the X-rays with the information being weighted by the exponential decrease of intensity versus depth [4, 7]. In case of using profile fitting methods asymmetric functions and the center of gravity of the fitted diffraction line shall be used.

$\mathrm{X}$-ray investigations on ceramics, shot peened with small shot sizes, show additional effects on the diffraction profiles. When the surface layer influenced by shot peening is significantly smaller than the penetration depth of the X-rays, a significant part of the diffraction profile is built up by the unstressed base material. Due to the generally small widths of diffraction lines of ceramics, a more or less clear separation of the contribution of the surface layer and the base material may be obtained. Using profile fitting techniques may allow to separately calculating the stress states of both the surface layer and the base material as well as the thickness of the surface layer.

\section{Effect of the stress gradients - penetration depths ratios on diffraction profiles}

The effect of the stress gradient with respect to the penetration depth is sketched in Fig. 1. If the penetration depth is less than the stressed surface layer (1), an asymmetric diffraction profile may 
occur. The location of the broadening of one flank of the diffraction line is depending on whether the near surface stress is compressive or tensile and is more or less continuous. In case of very steep stress gradients with the reversal point of the stress profile being close to the surface (2) and the thickness of the stressed surface layer being significantly smaller than the penetration depth of Xrays, the diffraction profile may show a splitting at one side of the profile. This is the situation handled in this paper.
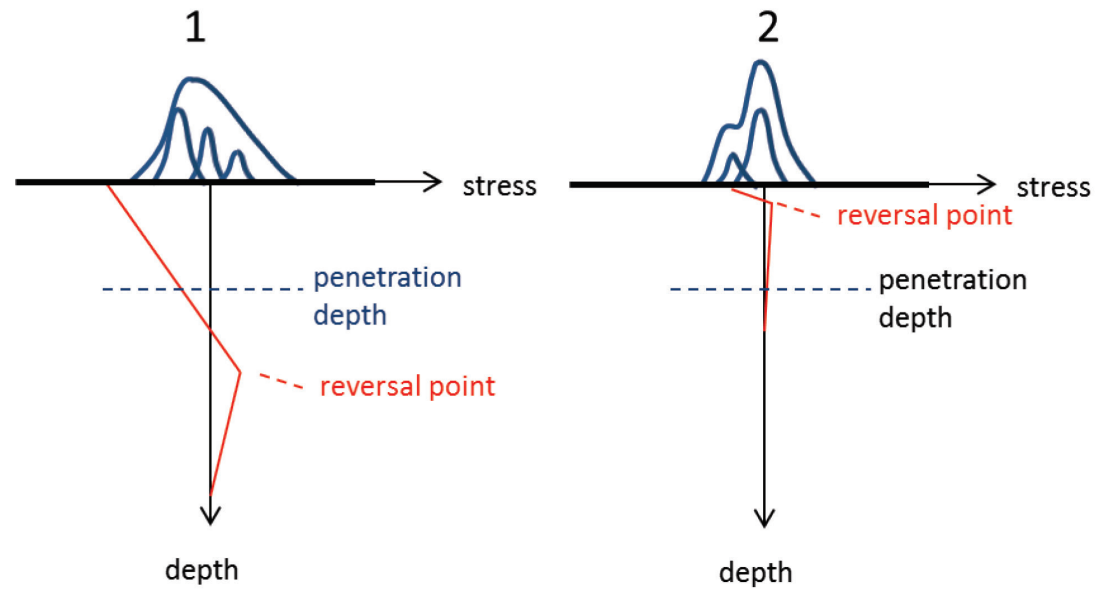

Figure 1: Sketch of diffraction lines resulting from different stress gradients - penetration depths ratios

\section{Experimental details}

Material. The material investigated was a commercially-available hot pressed silicon nitride ceramic ("HPSN-Black") fabricated by FCT Ingenieurkeramik GmbH, Germany. The most important material characteristic are a Young's modulus of $326 \mathrm{GPa}$, Poisson's ratio of 0.268, 4point bending strength of $900 \mathrm{MPa}$ and fracture toughness of $8 \mathrm{MPa} \cdot \mathrm{m}^{1 / 2}$. Stripes with a length $76 \mathrm{~mm}$, width of $19 \mathrm{~mm}$ and thicknesses of $0.9 \mathrm{~mm}$ were used.

Shot Peening. The shot peening was carried out with an injection system with gravimetric feeding. The pressurized air and the shot are applied to the jet nozzle in two different tubes. The shot is accelerated in the nozzle. The shots used were tungsten carbide beads with a diameter of $45 \mu \mathrm{m}-$ $135 \mu \mathrm{m}$ (mean $90 \mu \mathrm{m})$. The peening pressure was $0.3 \mathrm{MPa}$.

Determination of residual stresses. The diffraction profiles of $\{411\}$-lattice planes of $\beta$-silicon nitride occurring at $2 \theta \sim 125^{\circ}$ using $\mathrm{CrK} \alpha$-radiation were recorded for $15 \psi$-angles between $-56.8^{\circ}$ and $+56.8^{\circ}$ using a Bruker D8 in side inclination mode and a Braun position sensitive detector. A Xray elastic constant $1 / 2 \mathrm{~s}_{2}$ of $3.89 \mathrm{GPa}^{-1}$ was applied. The penetration depth from which $64 \%$ of the diffracted X-rays arose was $11 \mu \mathrm{m}$. A biaxial surface stress state with $\sigma_{33} \equiv 0 \mathrm{MPa}$ was assumed for fitting the resulting $\sin ^{2} \psi$ distributions of the lattice strains. Fitting and separation of the diffraction profiles, determination of the peak positions and calculation of stresses was done using an enhanced version of the Bruker software "STRESS", originally designed by Fraunhofer IWM.

Determination of layer thickness. From the integral intensities of separated diffraction lines the thickness $S_{\text {layer }}$ of the stressed surface layer can be calculated by Eq. 1:

$$
S_{\text {layer }}=\frac{\ln \frac{I_{\text {layer }}(\theta, \psi)}{I_{\text {layer }(\theta, \psi)}+I_{\text {substrate }(\theta, \psi)}}}{\mathbf{k}(\theta, \psi) \cdot \mu}
$$

with $I_{\text {layer }}=$ integral intensity of the diffraction line belonging to the stressed surface layer, $I_{\text {substrate }}=$ integral intensity of the diffraction line belonging to the non-stressed base material (substrate), $\mathrm{k}=$ factor counting for the penetration depth and $\mu=$ linear absorption coefficient. All these quantities 
are dependent on the tilt angle $\psi$ (and Bragg angle $\theta$ ). Thus, calculations for different tilt angles $\psi$ allow evaluating the scatter of results.

\section{Results}

Diffraction profiles. Fig. 2, left shows the $\{411\}-\mathrm{K} \alpha_{1+2}$ diffraction lines of the shot peened silicon nitride recorded at $15 \psi$-angles between $-56.8^{\circ}$ and $+56.8^{\circ}$. The asymmetry of the diffraction profiles and an indication of peak splitting in some cases can be seen. In Fig. 2, right, the diffraction profiles recorded at $\psi=-56.8^{\circ}$ and $\psi=0^{\circ}$ are shown. At $\psi=-56.8^{\circ}$ the slope of the right hand flank is less than the slope of the left hand flank. Together with the observation of a more or less continuous intensity course it can be concluded that the thickness of the stressed surface layer is in the range of the penetration depth (see clause 1, Fig. 1). At $\psi=0^{\circ}$ a significant shoulder (peak splitting) at the left hand flank of the diffraction profile can be obtained. This indicates the thickness of the stressed surface layer being less than the penetration depth (see clause 2, Fig. 1).
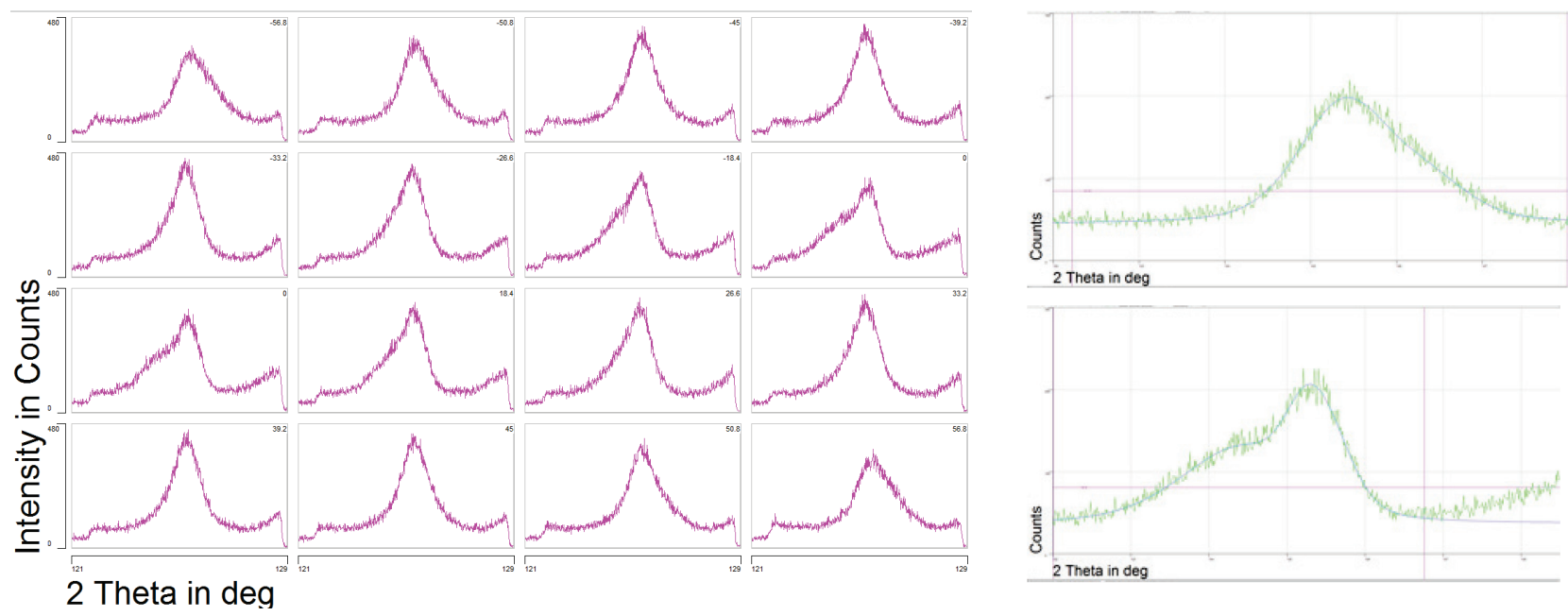

Figure 2: Left: $\{411\}-K \alpha_{1+2}$ diffraction lines of the shot peened silicon nitride recorded at $15 \psi$ angles between $-56.8^{\circ}$ and $+56.8^{\circ}$.

Right: Diffraction profile recorded at $\psi=-56.8^{\circ}$ (top) and $\psi=0^{\circ}$ (bottom), respectively, fitted by a Pseudo Voigt function doublet; displayed $2 \theta$-range is $122^{\circ}-128^{\circ}$, the vertical lines indicate the range of fitting.

Calculation of residual stresses. Calculation of residual stresses was performed using 3 different methods for the determination of peak positions: (i) the (sliding) center of gravity, (ii) peak fitting using the split Pearson Seven function, (iii) peak splitting using Pseudo Voigt functions.

(i) Center of gravity. A rough check whether the diffraction profiles are influenced by no-ideal diffraction situations, stress gradients and other physically caused circumstances is possible on basis of the sliding-center-of gravity method [1]. In this method the center of gravity is calculated using a gradually increasing intensity threshold. The result of this peak treatment (including background and Rachinger-K $\alpha_{2}$ subtraction) is shown in Fig. 3.

Remembering that the intensity threshold determines both the location and amount of the diffracting volume this quick check already indicates that at different depths different stress states are present. But the contribution of different depths to the diffraction profile cannot be concluded exclusively on the threshold as the peak intensities may be dominated by highly stressed surface layers or by a large volume with homogeneous stress below the surface. Nevertheless, from the slope of the $\sin ^{2} \psi$-distribution (see Fig. 4), increasing with higher $\sin ^{2} \psi$-values (reduced penetration depths, respectively), a higher amount of stress acting near the surface can be concluded. 

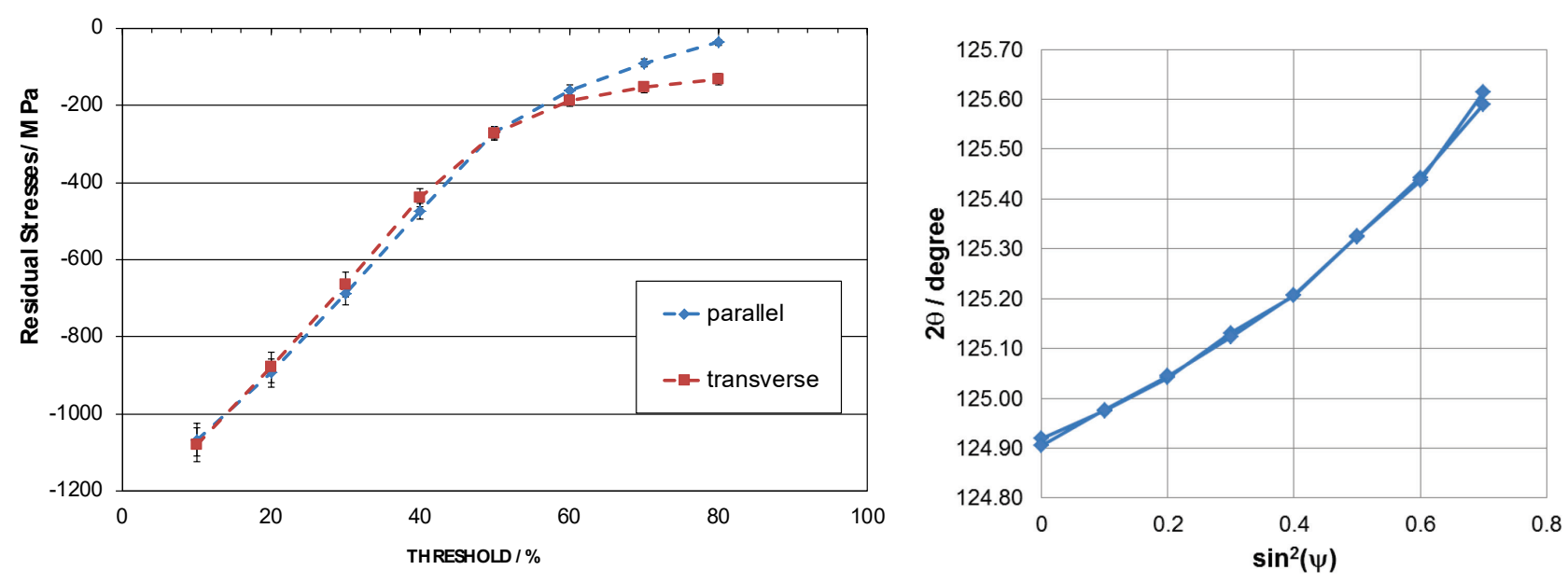

Figure 3: Dependency of the residual stress (parallel and transverse components) on the intensity threshold used for the calculation of the center of gravity of the diffraction line.

Figure 4: Diffraction angle $-\sin ^{2} \psi$ distribution of the shot peened specimen using the center of gravity and an intensity threshold of $10 \%$.

(ii) Split Pearson Seven. Peak fitting using a reasonable mathematical function is one of the most applied methods for determining the diffraction line position. Using more or less of the complete peak a statistically well covered result can be expected. Fitting asymmetric diffraction profiles needs asymmetric functions like the split Pearson Seven function consisting of two slightly shifted Pearson Seven functions applied to the flanks of the diffraction line. This may result in a good peak fit but the physical meaning behind that fit is somewhat questionable when using the $2 \theta$ - position at maximum intensity (which is the common procedure of commercial software). The slope of the resulting $\sin ^{2} \psi-$ distribution would indicate a tensile stress which would obviously not be expected for a shot peened surface layer. But, this agrees somewhat with the result of the center of gravity method (see Fig. 3) when using a high intensity threshold. In addition, the slope of the $\sin ^{2} \psi$-distribution, decreasing with increasing $\sin ^{2} \psi$ (decreasing penetration depth) indicates a tendency to smaller tensile stresses near the surface. Nevertheless, a stress calculation based on the Split Pearson Seven peak fit would lead to wrong results at all.

(iii) Peak splitting. Basis of this peak treatment was the assumption of shot peening using beads of only $90 \mu \mathrm{m}$ diameter would affect only a thin surface layer with a more or less stress free base material beneath. This assumption was confirmed qualitatively by the stress calculation using the sliding center of gravity method and the curvature of the $\sin ^{2} \psi$-distribution. The starting parameters for the separation of the diffraction profiles using two independent Pseudo Voigt functions -including the $K \alpha_{1}$ and $K \alpha_{2}$ diffraction lines were chosen from the peak with the most significant shoulder in one flank (see Fig. 2). 


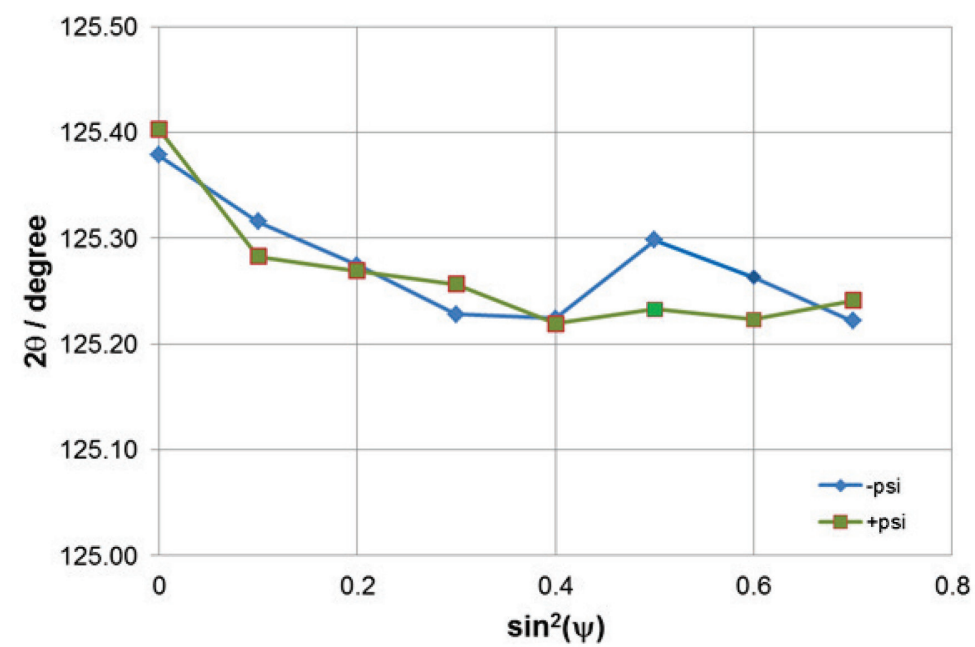

Figure 5: Diffraction angle $\sin ^{2} y$-distribution of the shot peened specimen using a split Pearson Seven fit. A linear regression of the distribution would indicate a tensile stress of $198 \pm 50 \mathrm{MPa}$.

In Fig. 6 the $\sin ^{2} \psi$-distributions resulting from the peak splitting procedure are shown. Both distributions show a more or less linear behavior indicating that no significant stress gradient could be resolved in the near surface and the base material. From the slopes a high compressive residual stress of $2936 \mathrm{MPa} \pm 118 \mathrm{MPa}$ can be calculated for the surface layer affected by shot peening; for the base material, not affected by shot peening, small tensile residual stresses of $125 \mathrm{MPa} \pm 8 \mathrm{MPa}$ are derived. Tensile residual stresses in the base material are needed to compensate compressive residual stresses in the surface layer.

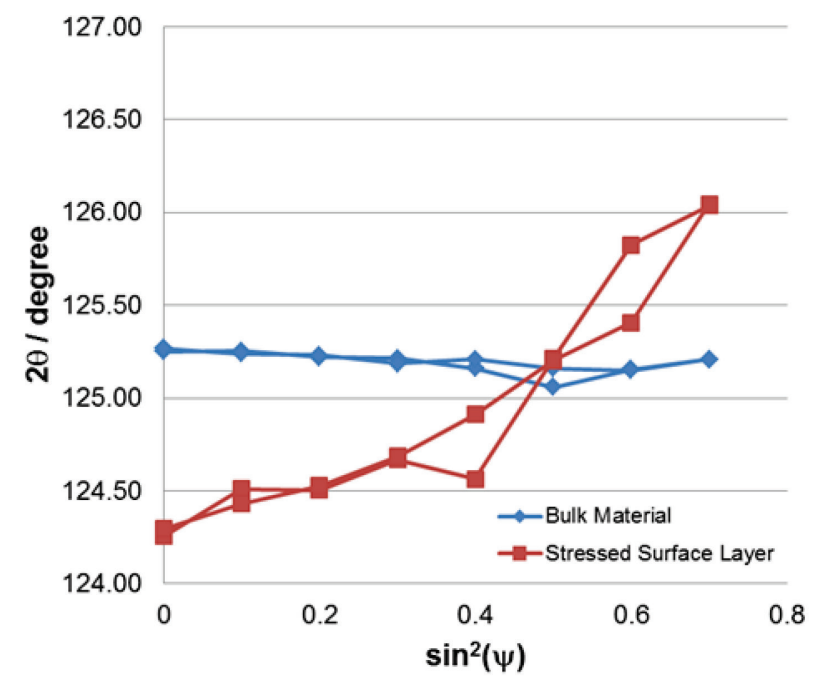

Fig. 6: Diffraction angle - $\sin ^{2} y$ distributions belonging to the stressed surface layer and the bulk material, respectively. The two distributions are derived from the peak splitting procedure.

\section{Thickness of the stressed surface layer}

Based on Eq. 1 the thickness of the stressed surface layer was calculated from two measurement directions phi $=0^{\circ}$ and $90^{\circ}$ (longitudinal and transverse). Due to the symmetry of the stress state resulting from shot peening, both measurements should give comparable values. This is confirmed by the results:

Phi $=0^{\circ}:$ Thickness of the stress surface layer $=4.95 \pm 1.05 \mu \mathrm{m}$

Phi $=90^{\circ}$ : Thickness of the stress surface layer $=4.94 \pm 0.7 \mu \mathrm{m}$ 


\section{Conclusion}

X-ray diffraction measurements were performed on silicon nitride ceramics shot peened with $90 \mu \mathrm{m}$ shot. The diffraction profiles show some asymmetry and in some cases evidence of peak splitting. The result of residual stress calculation is dramatically dependent on the method used to determine the diffraction line position. Fitting of distinct peaks to the intensity distributions leads to reasonable residual stress results for the surface layer influenced by the shot peening and the underlying base material. In addition, the thickness of the stressed surface layer can be calculated on basis of the intensities of the separated diffraction peaks. The results show, that the shot peening process led to extremely high compressive stresses in a very thin surface layer. The presented method may be applicable to all near surface stress states where the penetration depth is significantly larger than the thickness of the stressed surface layer.

\section{References}

[1] C. Genzel, Non-Destructive Analysis of Residual Stress Gradients $\sigma_{\mathrm{ij}}(\mathrm{z})$ in the Near-Surface Region by Diffraction Methods - Problems and Attempts at Their Solution, Proc. Of the 5th Intern. Conf. On Residual Stresses (1997) 514-521.

[2] B. Eigenmann, Non-Destructive and Partially Destructive Determination of Residual Stress State with Steep Stress Gradients, Advances in X-Ray Analysis 40 (1997).

[3] M. Härting, A Seminumerical Method to Determine the Depth Profile of the Three Dimensional Residual Stress State with X-Ray Diffraction, Acta Mater., Vol 40, No 4 (1998) 1427-1430. http://dx.doi.org/10.1016/S1359-6454(97)00284-X

[4] W. Pfeiffer, The role of the peak location method in X-ray stress measurement, Proc. of 4th International Conference on Residual Stresses; James, M.R. (Ed.); Society for Experimental Mechanics, Bethel, USA (1994) 148-155.

[5] M. Klaus, Röntgendiffraktometrische Ermittlung tiefenabhängiger

Eigenspannungsverteilungen in Dünnschichtsystemen mit komplexem Aufbau. Diss. Tu Berlin (2009).

[6] M. Klaus, et al., Application of energy-dispersive diffraction to the analysis of highly Inhomogeneous residual stress fields in thin film structures, Powder Diffraction 24 [Suppl.1] (2009), S82-S86. http://dx.doi.org/10.1154/1.3134362

[7] P.D. Evenschor, Zur röntgenographischen Ermittlung von Dehnungen beim Vorliegen von Dehnungsgradienten, Z. Metallkde. 74 (1983) 119. 